\title{
Determination of liquid-crystal polar anchoring energy by electrical measurements
}

\author{
Anatoli Murauski, Vladimir Chigrinov, Alexander Muravsky,* Fion Sze-Yan Yeung, Jacob Ho, and Hoi-Sing Kwok \\ Institute of Applied Physics Problems, Minsk, Belarus \\ and Hong Kong University of Science \& Technology, Clear Water Bay, Kowloon, Hong Kong
}

(Received 19 January 2005; published 21 June 2005)

\begin{abstract}
We propose a simple method for the determination of liquid-crystal (LC) polar anchoring energy by electrical measurements. The basic idea of this method is a two-channel scheme for capacitance measurements. The first channel uses one cell with a planar LC cell, while the second a LC cell with vertical alignment. One of the LC cells can have a high pretilt angle. The proposed method allows investigating anchoring properties of both planar and vertical aligned LC materials. Simultaneous measurements of the two cells compensate all volume effects in LC bulk and provide a good opportunity to study directly the LC-surface interaction. The method can be applied for LC cells, which do not have uniform azimuthal orientation. We used this method to investigate the polar anchoring properties of photoaligning material before and after illumination and for LC structures with a high pretilt angle.
\end{abstract}

DOI: 10.1103/PhysRevE.71.061707

PACS number(s): 64.70.Md

\section{INTRODUCTION}

The development of methods for anchoring energy measurements is especially important for low values of the anchoring strength, when the liquid-crystal (LC) alignment is not uniform or when the LC has a high pretilt angle on the surface alignment layers [1,2]. High-voltage measurements of the phase retardation usually require a high-quality antiparallel orientation in the LC cell [3-6]. Optical methods have certain problems in polar (zenithal) anchoring energy determination for vertically aligned LC cells [7-9]. In this case a capacitance technique can be applied [10]. We propose to use an additional LC cell with a perpendicular director orientation (planar or homeotropic) for the determination of the saturation level of the capacitance and for exclusion of certain volume effects, such as a variation of the LC order parameter under a high-field application $[11,12]$. Our technique can be successfully applied to both planar and vertically aligned LC materials. The calculation of the anchoring coefficient for materials with small pretilt angle can be done without additional information on the parameters of LC mixture (elastic and dielectric constants). Our method has a broad applicability as (i) the surface azimuthal energy need not be strong, (ii) the LC cell can have a high pretilt angle, (iii) the method is equally useful for measurements of polar anchoring energies in both planar and homeotropic cells, and (iv) the method applies to nematic LC's that have both a positive and negative dielectric anisotropy. Our method of LC polar anchoring measurements is expected to be helpful for a broad range of other homogeneous and tilted LC cells prepared by different techniques, such as rubbing [13], $\mathrm{SiO}$ oblique evaporation [14], atomic force microscope (AFM) pattern [15], or photoaligning [16].

\footnotetext{
*Present address: Belarussian State University, Minsk, Belarus.
}

\section{DETERMINATION OF LC POLAR ANCHORING ENERGY BY ELECTRICAL MEASUREMENTS}

The LC cell capacity is determined by the cell gap $d$, area of electrodes, $S$, and dielectric permittivity of $\mathrm{LC}, \varepsilon$, which is a function of the applied electric field. For high voltage $V$ $>6 V_{t h}$ the change of the capacity of the LC cell can be written in the following way $[4,5]$ :

$$
C=\frac{\varepsilon_{0} \varepsilon_{\|} S}{Q d}\left(1-\frac{\bar{V}}{V}\right),
$$

where $S$ is the electrodes overlapping square, $d$ is the cell thickness, $\varepsilon_{\|}$and $\varepsilon_{\perp}$ are the LC dielectric constants parallel and perpendicular to the LC director, and $\bar{V}=\alpha \varepsilon_{a} / \varepsilon_{\|} V_{t h}, \varepsilon_{a}$ $=\varepsilon_{\|}-\varepsilon_{\perp}, V_{t h}=\pi \sqrt{K_{1} / \varepsilon_{0} \varepsilon_{a}}$,

$$
\alpha=\frac{1}{\pi} \int_{y_{p}}^{1} \sqrt{\frac{(1+\gamma)(1+k y)}{y(1+\gamma y)}} d y,
$$

$\gamma=\left(\varepsilon_{\|}-\varepsilon_{\perp}\right) / \varepsilon_{\perp}, k=K_{3}-K_{1} / K_{1}, y=\sin ^{2} \theta, y_{p}=\sin ^{2} \theta_{p}, \theta_{p}$ the pretilt angle, $K_{1}$ and $K_{3}$ Frank elastic constants,

$$
\begin{gathered}
Q=1-\frac{2 \gamma K_{1}}{W d} B, \\
B=\frac{\left(1-k y_{p}\right)\left(1-y_{p}\right)}{\left(1-\gamma y_{p}\right)} .
\end{gathered}
$$

For the small pretilt angle $\theta_{p}<10^{\circ}$ we have $B \approx 1$. For the larger pretilt angle the coefficient $B$ can be calculated as a correction parameter.

Using Eq. (2) we can find the polar anchoring strength $W$ by measuring the value of the capacity $C_{\text {inf }}$ at a high-voltage $\left(V>V_{\text {th }}\right)$ limit for $V \rightarrow \infty$. The value of the threshold voltage $V_{\text {th }}$ can be found for the planar LC cell with a small pretilt angle in the case of a positive LC dielectric anisotropy $\varepsilon_{a}$ $=\varepsilon_{\|}-\varepsilon_{\perp}>0$ or a homeotropic (VA) cell in case of a negative dielectric anisotropy $\varepsilon_{a}<0$. For a positive liquid crystal, 


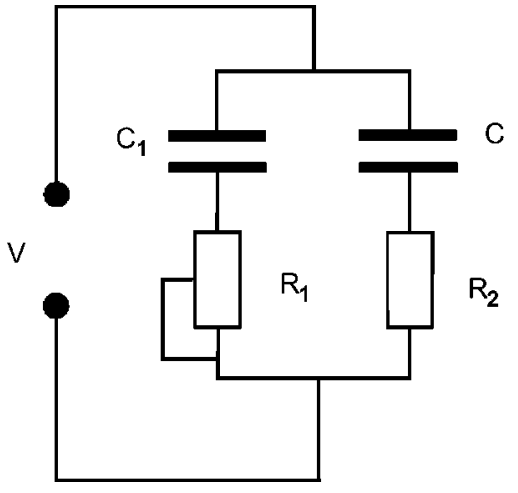

FIG. 1. Principal scheme for two-channel capacity measurements.

$$
W=\frac{2}{\pi^{2}} \frac{V_{\mathrm{th}}^{2}}{S} \frac{\left(C_{\|}-C_{\perp}\right)^{2}}{C_{\perp}} \frac{B}{1-C_{\|} / C_{\mathrm{inf}}},
$$

where $C_{\perp}$ and $C_{\|}$are the capacity of a LC cell for uniform planar $\left(\theta_{p}=0\right)$ and homeotropic orientation of LC cells, respectively. The thickness of the two LC cells must be equal. The relation (3) is a basic formula for the capacitance method.

The value $C_{\perp}$ can be obtained for a planar LC cell at the voltage $V<V_{\text {th }}$. We can also measure $C_{\text {inf }}$ in the limit of high voltages $V \rightarrow \infty$. At the same time the value of $C_{\|}$can be defined by applying a very high voltage, which reoriented LC molecules in LC bulk including molecules on the surface aligning layers. The basic problem of the capacity method is evaluating $C_{\|} / C_{\text {inf }}$.

To simplify the procedure of measurements and to avoid parasitic effects, such as an increase of the order parameter of the LC which takes place under high electric field (see the Appendix), we propose to define the relative capacity for planar and homeotropic cells simultaneously. The principal scheme of such measurement is shown in Fig. 1. Here ac voltage from a function generator is applied for the two channels at the same time. Cell 1 has a planar LC orientation and cell 2 homeotropic LC alignment. If we use a LC with a positive dielectric anisotropy, cell 1 will be switched in an electric field. Cell 2 will be used as a reference channel. Resistors $R_{1}$ and $R_{2}$ are used to measure the current at every channel. The resistance of LC cells $(>10 \mathrm{M} \Omega \mathrm{m})$ is much higher than the values of $R_{1}$ and $R_{2}(10 \mathrm{k} \Omega \mathrm{m})$, so we may consider only the capacitance of LC cells in the equivalent electrical circuit (Fig. 1). Resistor $R_{1}$ is a high-performance variable resistor. This resistor is used for the equalization of the two channels for the empty cells.

Expression (1) for the scheme shown in Fig. 1 can be written in the form

$$
\frac{U_{1}}{U_{2}} \frac{R_{2}}{R_{1}} \frac{C_{1}^{0}}{C_{2}^{0}}=\frac{1}{Q}\left(1-\frac{\bar{V}}{V}\right),
$$

where

$$
C=\frac{U_{1}}{V} \frac{1}{\omega R_{1}} \frac{C_{1}^{0}}{C_{2}^{0}}
$$

$$
C_{\|}=\frac{\varepsilon_{0} \varepsilon_{\|} S}{d}=\frac{U_{2}}{V} \frac{1}{\omega R_{2}},
$$

$\omega=2 \pi f, f$ the frequency of the applied voltage, $C_{1}^{0}$ and $C_{2}^{0}$ empty cell capacitance, and $U_{1}$ and $U_{2}$ the voltages on resistors $R_{1}$ and $R_{2}$ (Fig. 1 ).

The value of the resistor $R_{1}$ is defined as $\left(C_{1}^{0} / C_{2}^{0}\right) R_{2} / R_{1}$ $=1$ for the empty cells. After such a calibration formula (4) has the simplest form

$$
\frac{U_{1}}{U_{2}}=\frac{1}{Q}\left(1-\frac{\bar{V}}{V}\right)
$$

Expression (5) provides a simple way for the determination of the polar anchoring coefficient $W$. We must measure the dependence of $U_{1} / U_{2}$ on the applied voltage. Formula (5) is valid for $V>6 V_{\text {th }}$ and $V<V_{\max }$, where $V_{\max }$ is the maximal voltage, when a linear approximation for the function $\left(U_{1} / U_{2}\right)$ is possible. It corresponds to the condition, when the LC pretilt angle at the substrate is small enough $[4,5]$. The limit of the relation $\left(U_{1} / U_{2}\right)$ at $V \rightarrow \infty$ can be used for the calculation of the anchoring coefficient $W$ :

$$
W=\frac{2 \gamma K_{1}}{\left[1-\left(\frac{U_{1}}{U_{2}}\right)_{V \rightarrow \infty}\right] d}=\frac{2}{S}\left(\frac{V_{\mathrm{th}}}{\pi}\right)^{2}\left[1-\left(\frac{U_{1}}{U_{2}}\right)_{V \rightarrow \infty}\right],
$$

where $C_{\|}$and $C_{\perp}$ can be estimated from the experimental data,

$$
C_{\|}=\frac{U_{2}}{V} \frac{1}{R_{2} \omega}
$$

for any applied voltage,

$$
C_{\perp}=\frac{U_{1}}{V} \frac{1}{R_{1} \omega}
$$

for $V<V_{\mathrm{th}}$.

\section{LIQUID-CRYSTAL CELLS AND EXPERIMENTAL SETUP}

The increase of the measurement accuracy was achieved owing to the relative measurement of two equal cells with opposite orientation. The thickness of these cells must be stable and not change in the LC filling process. Thus the LC cell size has to be small and the glass substrates must be thick enough. We used cells with size $13 \times 13 \mathrm{~mm}^{2}$, width patch area $2 \mathrm{~mm}$, thickness of the glass substrate, $1.1 \mathrm{~mm}$, electrode area $5 \times 5 \mathrm{~mm}^{2}$, and cell gap $11.5 \mu \mathrm{m}$. The function generator AVTECH AV-151B-C, which had a maximal amplitude of the output signal of $200 \mathrm{~V}$, was used as an applied voltage source. For voltage measuring on resistors $R_{1}$ and $R_{2}$, an I/O board PCI-MIO-16E-4 from National Instruments was used. The value of resistors $R_{1}$ and $R_{2}$ was $10 \mathrm{k} \Omega$. The frequency of the applied signal was $1 \mathrm{kHz}$.

\section{RESULTS AND DISCUSSION}

The method was used to investigate the modification of anchoring properties of azodye photoalignment materials 


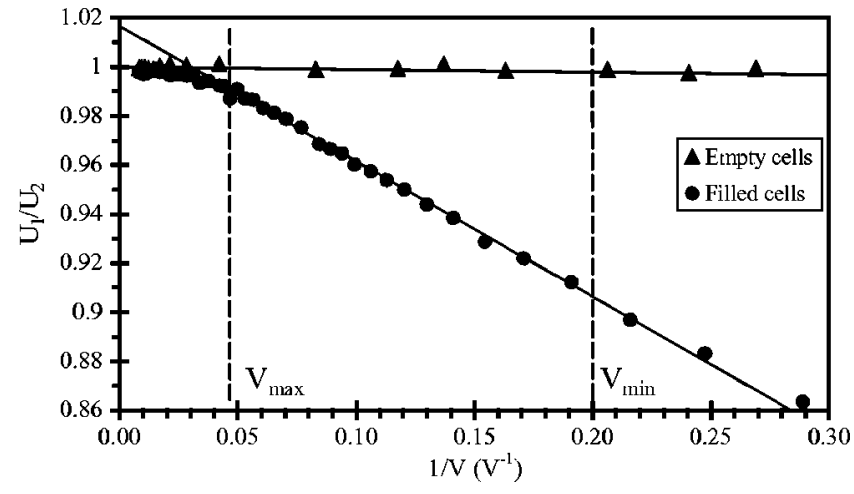

FIG. 2. Dependence of $U_{1} / U_{2}$ for LC cells with planar (cell 1, alignment material was SDA2+SD1) and homeotropic (cell 2, alignment material was JALC 2021) orientation on applied voltage $V$. Liquid crystal was MLC 5700-00. Thickness of the LC cells was $11.5 \mu \mathrm{m}$.

(mixture of SD1 and SDA2 [16,17]) for a various exposed radiation energy dose. Figure 2 shows the ratio $U_{1} / U_{2}$ in empty cells and cells filled with LC. The measured value of the threshold values for the cells was $V_{\text {th }}=0.8 \mathrm{~V}$, and we found that optimal voltage range for linear approximation was obtained at $V_{\min }=5 \mathrm{~V}$ and $V_{\max }=20 \mathrm{~V}$. We found $W_{1}$ $=3.81 \times 10^{-4} \mathrm{~J} / \mathrm{m}^{2}$ for the polar anchoring coefficient before the exposure and $W_{2}=3.44 \times 10^{-4} \mathrm{~J} / \mathrm{m}^{2}$ after an exposed dose of $0.8 \mathrm{~W} / \mathrm{cm}^{2}$. This result means that the photoalignment material SDA2+SD1 does not exhibit any change of polar anchoring energy versus exposure time. For high voltage $(120 \mathrm{~V})$ we received a saturation value of $U_{1} / U_{2}$. The saturation value was very close to 1 , which is expected for two cells with equal thickness. When the pretilt angle $\theta_{p}$ $>10^{\circ}$, the coefficient $B$ in Eq. (2) decreases, which must be allowed for (Fig. 3).

We also measured the polar anchoring energy for a high pretilt angle of the LC director in the cell, simultaneously using two polyimide (PI) agents with different aligning capability: PI for homeotropic and PI for planar LC alignment [18]. Using different concentrations of homeotropic PI in planar PI (from $1 \%$ to $10 \%$ ), we received a LC cell with different pretilt angles and measured the corresponding polar

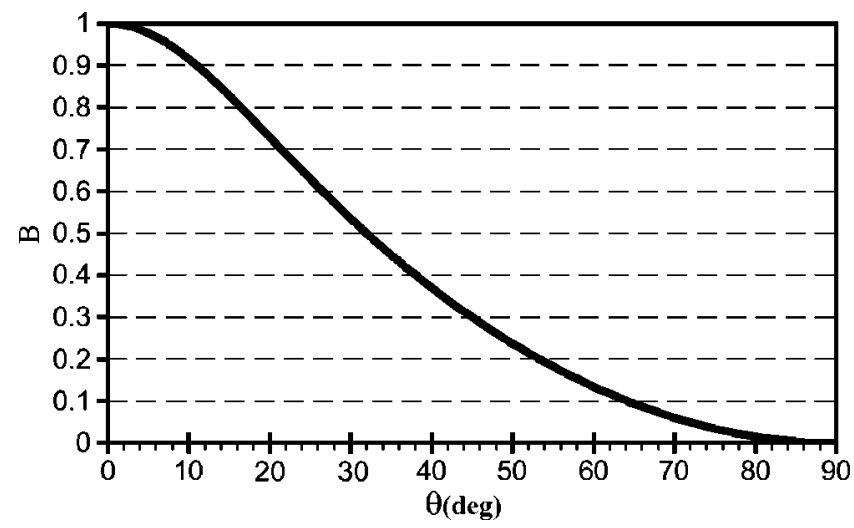

FIG. 3. Calculated dependence of the correction parameter $B$, Eq. (2), versus pretilt angle. The parameter $\left(K_{3}-K_{1}\right) / K_{1}=1,\left(\varepsilon_{\|}\right.$ $\left.-\varepsilon_{\perp}\right) / \varepsilon_{\perp}=3$.

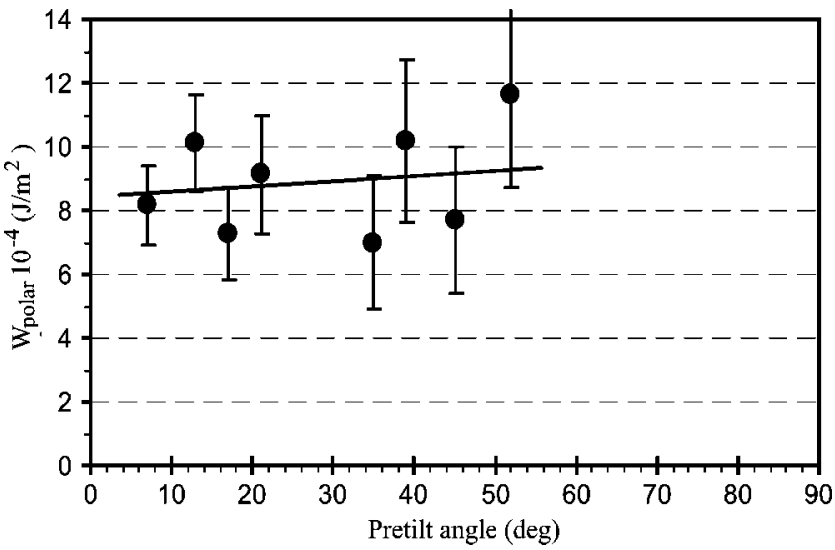

FIG. 4. Polar anchoring energy dependence on pretilt angle for high pretilt substrates, prepared with planar and homeotropic aligning layers.

anchoring energy (Fig. 4). We found that the LC polar energy is almost independent of the value of the pretilt angle, which seems reasonable [19]. The value of the polar anchoring energy was about $0.8-1 \times 10^{-3} \mathrm{~J} / \mathrm{m}^{2}$ within the whole range of the LC pretilt angles from $\theta_{p}=2^{\circ}$ to $\theta_{p}=66^{\circ}$ (Fig. 4).

\section{CONCLUSION}

We propose a modification of the capacitance method for the LC polar anchoring energy measurements. The proposed method is simple and increases the accuracy of the capacity method for the determination of the polar anchoring energy, especially for nonuniform azimuthal LC alignment, and can be used for alignment material with a high pretilt angle. This technique can be used for the investigation of both planar and homeotropic-aligned materials and for LC having both positive and negative dielectric anisotropy without any modification of the experimental scheme. If aligning materials provide a small pretilt angle $\theta_{p}<10^{\circ}$, the polar anchoring coefficient can be defined without using the LC materials parameters (elastic and dielectric constants). In the case of a high pretilt angle we should know additional information about the elastic ratio $K_{3} / K_{1}$ for the correction coefficient. The polar anchoring energy of the photoaligned azodye layers was found to be $3.8 \times 10^{-4} \mathrm{~J} / \mathrm{m}^{2}$, almost independent of the UV exposure energy. We also measured the polar anchoring energy for a high pretilt angle of the LC director in the cell, simultaneously using two polyimide (PI) agents with different aligning capability, and found that the polar anchoring energy is almost independent of the pretilt angle.

\section{ACKNOWLEDGMENTS}

This research was partially supported by ITF Grant No. ITS/111/03 and RGC Grant Nos. HKUST6102/03E and HKUST6149/04E.

\section{APPENDIX}

The effect of the increase of the nematic LC bulk order [11] including the formation of a biaxial nematic by chang- 


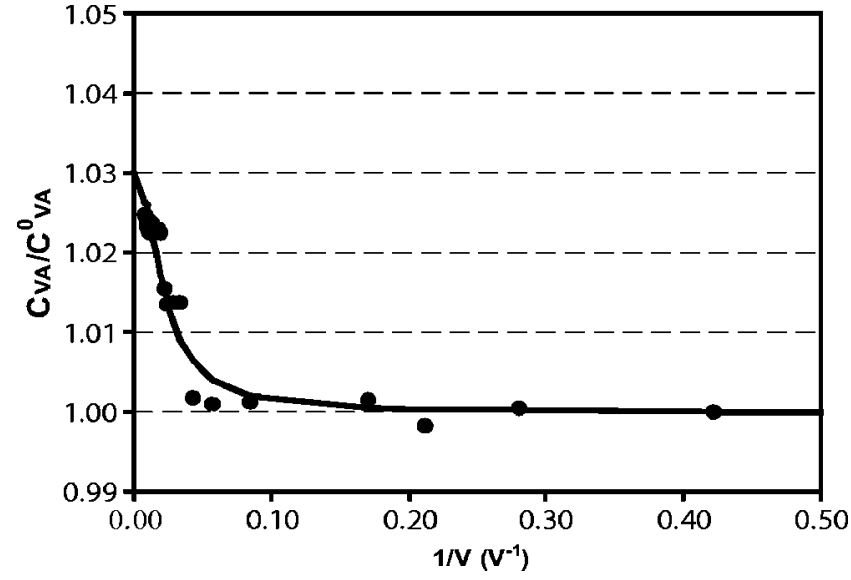

FIG. 5. Relative change of the capacity VA LC cell $C_{\mathrm{VA}} / C_{\mathrm{VA}}^{0}$ in a high electric field. The cell was filled with MLC 5700-000, having a positive dielectric anisotropy. The cell thickness was $11 \mu \mathrm{m}$. The PI aligning agent JALC-2021 was used to provide a homeotropic LC alignment.

ing the order parameter in high electric [10] or magnetic fields [20] has been already discussed. The value of the electric fields $E_{\mathrm{S}}$, when such effects become possible, can be roughly estimated by comparing the electric field coherence length $\xi_{\mathrm{E}} \approx 1 / E \sqrt{K_{1} / \varepsilon_{0} \varepsilon_{a}}$ with a characteristic thickness of the order parameter variation from the bulk to the surface value, which is few molecular lengths $\xi_{\mathrm{s}} \approx 10-100 \mathrm{~nm}[21]$. Comparing $\xi_{\mathrm{E}} \approx \xi_{\mathrm{s}}$ we get the typical values of $E_{\mathrm{S}}$ $\approx 10^{5}-10^{6} \mathrm{~V} / \mathrm{cm}$.

If the capacitance method is applied directly, we may observe the above-mentioned increase of the order parameter of the LC cell due to the ordering of the LC layer under high voltage and consequently the variation of the LC cell capacitance. The effect of the capacitance increase can be observed if we apply a high voltage to a homeotropic LC cell. The relative change of the capacitance (or LC dielectric constant) is shown in Fig. 5. As followed from Fig. 5, the orderparameter-induced change of the capacitance is about $2 \%$, while for an accurate definition of the polar anchoring energy we need to measure an capacitance with an accuracy of $0.1 \%$. As a result of the order parameter variation, the saturation of the capacitance for the planar LC cell may not be observed at high electric field and the ratio $C_{\|} / C_{\text {inf }}$ cannot be properly obtained from the experiment.

The second problem of the capacitance method is the influence of the thickness of the aligning dielectric layer on the measured values of the LC capacitance. If the thickness of the aligning layer is not properly fixed, the error increases and the results become not reproducible. We used the following simple formula for the capacitance $C$ in our calculations:

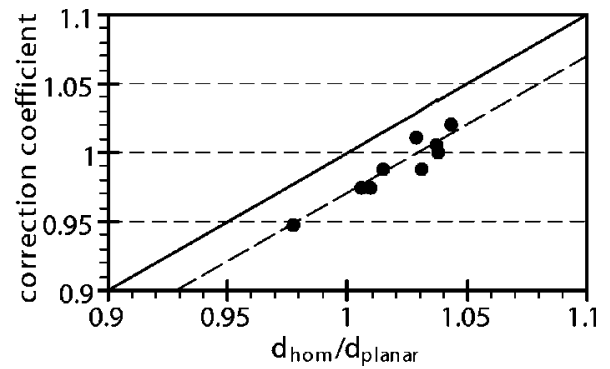

FIG. 6. Correction coefficient versus the ratio of the cell gaps of homeotropic to planar LC cells $d_{\text {hom }} / d_{\text {planar }}$. Dots indicate the correction parameter versus $d_{\text {hom }} / d_{\text {planar }}$ received in experiment. Solid line shows the correction parameter when the thickness of the aligning layers $d_{a l}$ in homeotropic and planar LC cells is equal. Dashed line is the approximation of the experimental data.

$$
C=\frac{\varepsilon_{0} S}{\frac{d_{L C}}{\varepsilon_{L C}}+\frac{2 d_{a l}}{\varepsilon_{a l}}},
$$

where $d_{L C}, d_{a l}$ and $\varepsilon_{L C}, \varepsilon_{a l}$ are the thickness and dielectric constants of the LC and aligning layers, respectively, and the $S$ surface area of the LC cell. According to Eq. (A1) the variation of the aligning layer thickness from 50 to $100 \mathrm{~nm}$ leads to the variation of the LC capacitance by $4 \%-8 \%$. Thus the information on the limited values of the capacitance at a high electric field becomes inaccurate and we may face large errors in LC polar anchoring energy evaluation by the capacitance method. To increase the accuracy of the capacitance method we have to introduce the correction, which provides the saturation value for $U_{1} / U_{2}$ equal to 1 (Fig. 6). The measurements were performed in the following way: (i) the ratio of the capacitance of the two empty cells was measured; (ii) after filling the cells with LC two different LC cells were formed with homeotropic and planar LC alignment; (iii) high voltages $(100-120 \mathrm{~V})$ were applied to the cells and the capacitance ratio was measured in the approximation of infinite fields. If the thickness of the aligning layers of the two LC cells is the same, then the two capacitance ratios measured at the steps (i) and (iii) should be the same. However, in reality these ratios are different, and consequently, the correction should be introduced to provide the saturation point for $U_{1} / U_{2}$ equal to 1 by subsequent multiplication of all experimental values of $U_{1} / U_{2}\left(V^{-1}\right)$ by the same correction coefficient (Figs. 2 and 6).

Our method of the simultaneous two-LC-cell capacitance measurements allows solving all the above-mentioned problems and getting reliable values of the LC polar anchoring values by the capacitance method. Figure 2 clearly demonstrates the measurement results. 
[1] D. Subacius, V. M. Pergamenshchik, and O. D. Lavrentovich, Appl. Phys. Lett. 67, 214 (1995).

[2] R. W. Fitas and S. P. Patel, Appl. Phys. Lett. 50, 1426 (1987).

[3] H. Yokoyama and H. A. van Sprang, J. Appl. Phys. 57, 4520 (1985).

[4] Yu. A. Nastishin, R. D. Polak, S. V. Shiyanovskii, V. H. Bodnar, and O. D. Lavrentovich, J. Appl. Phys. 86, 4199 (1999).

[5] Yu. A. Nastishin, R. D. Polak, S. V. Shiyanovskii, and O. D. Lavrentovich, Appl. Phys. Lett. 75, 202 (1999).

[6] D. Shenoy, L. Beresnev, D. Holt, and R. Shashidhar, Appl. Phys. Lett. 80, 1538 (2002).

[7] J. G. Fonseca and Y. Galerne, Phys. Rev. E 61, 1550 (2000).

[8] M. Vilfan and M. Copic, Phys. Rev. E 68, 031704 (2003).

[9] H. Yokoyama and R. Sun, Jpn. J. Appl. Phys., Part 2 39, L45 (2000).

[10] Y. Toko and T. Akahane, Mol. Cryst. Liq. Cryst. Sci. Technol., Sect. A 368, 469 (2001).

[11] P. Martinot-Lagarde, H. Dreyfus-Lambez, and I. Dozov, Phys. Rev. E 67, 051710 (2003).

[12] I. Lelidis, M. Nobili, and G. Durand, Phys. Rev. E 48, 3818 (1993).
[13] B. R. Acharya, J. H. Kim, and S. Kumar, Phys. Rev. E 60, 6841 (1999).

[14] I. Dozov and Ph. Martinot-Lagarde, Phys. Rev. E 58, 7442 (1998).

[15] A. Rastegar, M. Skarabot, B. Blij, and Th. Rasing, J. Appl. Phys. 89, 960 (2001).

[16] V. Chigrinov, S. Pikin, A. Verevochnikov, V. Kozenkov, M. Khazimullin, J. Ho, D. D. Huang, and H. S. Kwok, Phys. Rev. E 69, 061713 (2004).

[17] H. Takada, H. Akiyama, H. Takatsu, V. Chigrinov, E. Prudnikova, V. Kozenkov, and H. S. Kwok, SID’03 Digest, 620 (2003).

[18] F. Yeung, F. Xie, J. Wan, O. Tsui, P. Sheng, and H. S. Kwok, SID’05 Digest, 1080 (2005).

[19] V. G. Chigrinov, Liquid Crystal Devices: Physics and Applications (Artech-House, Boston, 1999).

[20] B. Malraison, Y. Poggi, and E. Guyon, Phys. Rev. A 21, 1012 (1980).

[21] P. Sheng, B. Z. Li, M. Zhou, T. Moses, and Y. R. Shen, Phys. Rev. A 46, 946 (1992). 